\title{
A NOTE ON MAXIMAL ESTIMATES FOR STOCHASTIC CONVOLUTIONS
}

\author{
MARK VERAAR AND LUTZ WEIS
}

\begin{abstract}
In stochastic partial differential equations it is important to have pathwise regularity properties of stochastic convolutions. In this note we present a new sufficient condition for the pathwise continuity of stochastic convolutions in Banach spaces.
\end{abstract}

\section{INTRODUCTION AND MAIN RESULT}

Let $(\Omega, \mathcal{A}, \mathbb{P})$ be a probability space with filtration $\left(\mathscr{F}_{t}\right)_{t \geq 0}$. Let $(S(t))_{t \geq 0}$ be a strongly continuous semigroup on a Banach space $X$. We will be interested in obtaining conditions for path-continuity of the stochastic convolution

$$
S \diamond G(t):=\int_{0}^{t} S(t-s) G(s) d W_{H}(s),
$$

where $G: \mathbb{R}_{+} \times \Omega \rightarrow \mathscr{L}(H, X)$ is such that the stochastic integral with respect to the cylindrical Brownian motion $W_{H}$ exists. There are many such continuity results in the literature (see [4, 6, 9, 16, 17, 27, and references therein).

Our methods to obtain continuity results are based on techniques similar to the ones in 6, 16, 17. The results are comparable with 6] but are of independent interest. The methods we present can also be applied for other stochastic convolutions $\int_{0}^{t} S(t-s) d M(s)$, where $M$ is an $X$-valued local martingale, as soon as one has a "decent" stochastic integration theory for integration with respect to $M$. For instance, some of our methods can also be applied in the case $W_{H}$ is replaced by a continuous local martingale (see [36]) or a Lévy process (cf. [5]). In this paper this has not been considered and we leave this to the interested reader.

For $\sigma \in(0, \pi)$ let

$$
\Sigma_{\sigma}:=\{\lambda \in \mathbb{C} \backslash\{0\}: \arg (\lambda)<\sigma\}
$$

denote the open sector of angle $\sigma$ in the complex plane. A closed and densely defined operator $A$ on $X$ is sectorial of type $\phi \in[0, \pi)$ if $A$ is one-to-one with dense range and for all $\sigma \in(\phi, \pi)$ we have $\Sigma_{\sigma} \subseteq \varrho(A)$ and

$$
\sup _{\lambda \in \Sigma_{\sigma}}\|\lambda R(\lambda, A)\|<\infty \text {. }
$$

1991 Mathematics Subject Classification. Primary: 60H15; Secondary: 35B65, 35R60, 46B09, $47 \mathrm{D} 06$.

Key words and phrases. stochastic convolutions, maximal inequalities, path-continuity, stochastic partial differential equations, $H^{\infty}$-calculus, $\gamma$-radonifying operators, exponential tail estimates.

The first author was supported by a VENI subsidy 639.031.930 of the Netherlands Organization for Scientific Research (NWO). The second named author is supported by a grant from the Deutsche Forschungsgemeinschaft (We 2847/1-2). 
Here, $R(\lambda, A):=(\lambda-A)^{-1}$.

We introduce the following condition on a sectorial operator $A$.

$(H)$ The operator $-A$ has a bounded $H^{\infty}$-calculus of angle $<\pi / 2$.

For details on $H^{\infty}$-calculus for sectorial operators we refer the reader to [15, 20, 24, 37. The condition $(H)$ implies that $A$ generates an analytic semigroup $S(t)=e^{t A}$. Many differential operators on $L^{q}$-spaces with $q \in(1, \infty)$ which generate an analytic semigroup satisfy condition $(H)$. We will show how one can use condition $(H)$ to obtain a continuity result for stochastic convolutions. Below we present several situations which are not covered by the existing literature. The existing results always require that the semigroup is contractive or quasi-contractive. Recall that $S(t)$ is called quasi-contractive if there exists a $w \in \mathbb{R}$ such that for all $t \geq 0$, $\|S(t)\| \leq e^{w t}$.

The next theorem is our first main result. It will be formulated for UMD Banach spaces $X$ with type 2 . Recall that $X=L^{q}$ with $q \in[2, \infty)$ is an example of a UMD space with type 2 . Moreover, every space which is isomorphic to a closed subspace of $L^{q}$ with $q \in(1, \infty)$ is UMD and of type 2. For UMD spaces with type 2 a class of stochastically integrable processes is given by the adapted and strongly measurable processes $G$ for which $G \in L^{2}\left(\mathbb{R}_{+} ; \gamma(H, X)\right)$ almost surely (see Proposition 2.1 for details). The set of all adapted $G$ which are in $L^{2}\left(\mathbb{R}_{+} ; \gamma(H, X)\right)$ is denoted by $L_{\mathscr{F}}^{0}\left(\Omega ; L^{2}\left(\mathbb{R}_{+} ; \gamma(H, X)\right)\right)$. For details on the space of $\gamma$-radonifying operators $\gamma(H, X)$ we refer to [25].

Theorem 1.1. Let $X$ be a UMD space with type 2. Assume A satisfies hypothesis $(H)$. Then for all $G \in L_{\mathscr{F}}^{0}\left(\Omega ; L^{2}\left(\mathbb{R}_{+} ; \gamma(H, X)\right)\right)$ the process $S \diamond G$ has a version with continuous paths. Moreover, for all $p \in(0, \infty)$, the following maximal estimate holds:

$$
\left(\mathbb{E} \sup _{t \geq 0}\|S \diamond G(t)\|^{p}\right)^{1 / p} \leq C_{1} C_{2}\left(\mathbb{E}\|G\|_{L^{2}\left(\mathbb{R}_{+} ; \gamma(H, X)\right)}^{p}\right)^{1 / p},
$$

where $C_{1}$ depends on $A$, and $C_{2}$ depends on $X$ and $p$.

As a corollary by an easy translation argument one can prove a version on bounded intervals $[0, T]$ in the case where only $A-w$ satisfies hypothesis $(H)$ for some $w>0$. This gives an extra exponential factor $e^{w T}$.

The proof of Theorem 1.1 will be given in Section 2 and uses a dilation argument in a similar spirit as [16, 17. However, the enlargements of the spaces we need to consider are more complicated. After the first version of this paper was written we found out that Theorem 1.1 was also proved by Seidler in the setting $X=L^{q}$ with $q \in[2, \infty)$ (see [33]).

Under different conditions on the Banach space (see Section 3) it was proved in [6] that for every $A$ for which $S(t)$ is (quasi)-contractive, there exists a continuous version of $S \diamond G$. Our result is not covered by this result since there are many interesting examples of differential operators $A$ which satisfy $(H)$, but for which $S(t)$ is not quasi-contractive, or not known to be quasi-contractive. For instance in [21] it was proved that semigroups generated by differential operators of order higher than two, are never contractive. Moreover, second order differential operators with irregular coefficients are often not quasi-contractive (see [23, Theorems 1.1 and 1.2]). Except for trivial cases, almost no positive results on quasi-contractiveness of semigroups generated by systems of differential operators are known. The only (easy 
to describe) class of scalar differential operators which generate an analytic semigroups which is quasi-contractive, seems to be second order differential operators in divergence form with smooth coefficient. Already in 2] it was shown that under fairly general boundary conditions these operators generate a quasi-contractive semigroup.

On the other hand, 6] can be applied for instance to translation semigroups on $L^{p}$ with $p \in[2, \infty)$. This is not covered by Theorem 1.1 because condition $(H)$ implies analyticity of the semigroup. In Section 3 we present an alternative approach to obtain Theorem 1.1 with slightly different assumptions on the Banach space $X$.

The following frequently arising examples in applications are not covered by the continuity theorem in [6].

Example 1.2. Let $q \in(1, \infty)$. Let $A$ be a system of second order operators on a $C^{2}$-domain $\mathcal{O} \subset \mathbb{R}^{n}$ :

$$
(A f)(x)=\sum_{i, j=1}^{n} a_{i j}(x) D_{i} D_{j} f(s)+\sum_{i=1}^{n} b_{i}(x) D_{i} f(x)+c(x) f(x)
$$

with Dirichlet boundary conditions. Let $X=L^{q}(\mathcal{O})$. If the $a_{i j} \in C^{\epsilon}\left(\overline{\mathcal{O}} ; \mathbb{C}^{N \times N}\right)$ are uniformly parameter elliptic on $\overline{\mathcal{O}}$, and $b_{i} \in L^{\infty}\left(\mathcal{O} ; \mathbb{C}^{N \times N}\right)$ and $c \in L^{\infty}\left(\mathcal{O} ; \mathbb{C}^{N \times N}\right)$, then $A-w$ satisfies $(H)$ (see [11]) for some $w \in R$ large enough. Therefore, if $q \in[2, \infty)$, then Theorem 1.1 is applicable. However, $S(t)$ is not known to be quasi-contractive in this general situation.

The example can also be extended to systems of higher order elliptic operators as long as the Lopatinskii-Shapiro conditions hold (see [11). As we already said before the semigroups generated by such higher order operators are never contractive.

In Section 4 we prove another result on the path-continuity of $S \diamond G$. Here we assume less on the Banach space $X$ and on the processes $G$. The result there covers all $L^{q}$-spaces with $q \in(1, \infty)$.

\section{Proof of Theorem 1.1}

Before, we give the proof of the theorem recall the following result on stochastic integration theory. We refer to [4] and [26] for details.

Proposition 2.1. Assume $X$ is a UMD Banach space with type 2 . If $G: \mathbb{R}_{+} \times$ $\Omega \rightarrow \gamma(H, X)$ is adapted and strongly measurable and $G \in L^{2}\left(\mathbb{R}_{+} ; \gamma(H, X)\right)$ a.s., then $G$ is stochastically integrable with respect to $W_{H}$, and the $X$-valued process $t \mapsto \int_{0}^{t} G(s) d W_{H}(s)$ is an a.s. pathwise continuous local martingale. Moreover, for all $p \in(0, \infty)$ one has

$$
\mathbb{E}\left(\sup _{t \geq 0}\left\|\int_{0}^{t} G(s) d W_{H}(s)\right\|^{p}\right) \leq C_{p, X}^{p}\|G\|_{L^{p}\left(\Omega ; L^{2}\left(\mathbb{R}_{+} ; \gamma(H, X)\right)\right)}^{p},
$$

where $C_{p, X}$ depends only on $p$ and $X$.

The case $p \leq 1$ was not considered in [4] or [26, but can easily be obtained by an application of Lenglart's inequality (see [22]).

Remark 2.2. Recently, in [33] Seidler found the optimal asymptotic behavior of $C_{p, X}$ from (2.1). Using the result of [29] he showed that if $X$ is 2-smooth, then there exists a constant $C_{X}$ such that $C_{p, X} \leq C_{X} \sqrt{p}$ for $p \geq 2$. This result applies 
to our setting in Proposition 2.1. since UMD spaces with type 2 have martingale type 2 (see [3]) and such spaces can be renormed such that their norm is 2-smooth (see [30]). An alternative proof of the behavior of the constant in the setting of $L^{q}$-spaces based on interpolation, can be found in Corollary A.4 below.

Lemma 2.3. For a Banach space $X$ the following assertions hold:

(1) If $X$ has type 2 , then also the space $\gamma\left(L^{2}\left(\mathbb{R}_{+} ; H\right), X\right)$ has type 2.

(2) If $X$ has $U M D$, then also the space $\gamma\left(L^{2}\left(\mathbb{R}_{+} ; H\right), X\right)$ has $U M D$.

Proof. It is well-known that the assertion holds if the space $\gamma\left(L^{2}\left(\mathbb{R}_{+} ; H\right), X\right)$ is replaced by $L^{2}(\Omega ; X)$. Now the result follows since $\gamma\left(L^{2}\left(\mathbb{R}_{+} ; H\right), X\right)$ is isometric to a closed subspace of $L^{2}(\Omega ; X)$, and therefore inherits the Banach spaces properties type 2 and UMD.

Proof of Theorem 1.1, Let $Y=\gamma\left(L^{2}(\mathbb{R}), X\right)$. By [13] the boundedness of the $H^{\infty}$ calculus with angle $<\pi / 2$, yields the following dilation result:

There are $J \in \mathscr{L}(X, Y), P \in \mathscr{L}(Y)$ and $(U(t))_{t \in \mathbb{R}}$ in $\mathscr{L}(Y)$ such that:

(i) There are $c, C \geq 0$ such that for all $x \in X$, one has $c\|x\| \leq\|J x\|_{Y} \leq C\|x\|$.

(ii) $P$ is a projection onto $J(X)$.

(iii) $(U(t))_{t \in \mathbb{R}}$ is a strongly continuous group on $Y$ with $\|U(t) y\|_{Y}=\|y\|_{Y}$ for all $y \in Y$.

(iv) For all $t \geq 0$ one has $J S(t)=P U(t) J$.

Clearly, we have

$$
J S \diamond G(t)=\int_{0}^{t} J S(t-s) G(s) d W_{H}(s)=P U(t) \int_{0}^{t} U(-s) J G(s) d W_{H}(s),
$$

To see that the latter stochastic integral exists in $Y$, note that $s \mapsto U(-s) J G(s)$ is strongly measurable and adapted, and

$$
\|U(-s) J G(s)\|_{L^{2}\left(\mathbb{R}_{+} ; \gamma(H, Y)\right)} \leq C\|G\|_{L^{2}\left(\mathbb{R}_{+} ; \gamma(H, X)\right)}<\infty \text { a.s. }
$$

Since $Y$ has UMD and type 2 by Lemma2.3, it follows from Proposition 2.1 that $t \mapsto$ $\int_{0}^{t} U(-s) J G(s) d W_{H}(s)$ exists and has a version which is a.s. pathwise continuous. Therefore, by (2.2) and the strong continuity of $U(t)$ it follows that $J S \diamond G$ has a version which is a.s. pathwise continuous. By (i) also $S \diamond G$ has a version which is a.s. pathwise continuous. Moreover, if $G \in L^{p}\left(\Omega ; L^{2}\left(\mathbb{R}_{+} ; \gamma(H, X)\right)\right)$ using (2.2) and Proposition 2.1 one obtains the following estimate

$$
\begin{aligned}
\mathbb{E}\left(\sup _{t \geq 0}\|S \diamond G(t)\|^{p}\right) & \leq c^{-p} \mathbb{E}\left(\sup _{t \geq 0}\|J S \diamond G(t)\|_{Y}^{p}\right) \\
& =c^{-p} \mathbb{E}\left(\sup _{t \geq 0}\left\|P U(t) \int_{0}^{t} U(-s) J G(s) d W_{H}(s)\right\|_{Y}^{p}\right) \\
& \leq c^{-p}\|P\|^{p} \mathbb{E}\left(\sup _{t \geq 0}\left\|\int_{0}^{t} U(-s) J G(s) d W_{H}(s)\right\|_{Y}^{p}\right) \\
& \leq c^{-p}\|P\|^{p} C_{p, Y}^{p}\|U(-s) J G(s)\|_{L^{p}\left(\Omega ; L^{2}\left(\mathbb{R}_{+} ; \gamma(H, Y)\right)\right)}^{p} \\
& \leq c^{-p}\|P\|^{p} C_{p, Y}^{p} C^{p}\|G\|_{L^{p}\left(\Omega ; L^{2}\left(\mathbb{R}_{+} ; \gamma(H, X)\right)\right)}^{p}
\end{aligned}
$$

This completes the proof of (1.1) with $C_{1}=c^{-1} C\|P\|$ and $C_{2}=C_{p, Y}$. 
It is natural to ask whether one also has exponential estimates (cf. [6, 17] and references therein) for $\sup _{t \geq 0}\|S \diamond G(t)\|$. This is indeed the case as follows from the next result.

Theorem 2.4. Assume $X$ is a UMD Banach space with type 2. Assume condition $(H)$ holds. If $G \in L_{\mathscr{F}}^{0}\left(\Omega ; L^{2}\left(\mathbb{R}_{+} ; \gamma(H, X)\right)\right)$ is such that for some $M>0$, almost surely

$$
\|G\|_{L^{2}\left(\mathbb{R}_{+} ; \gamma(H, X)\right)} \leq \sqrt{M}
$$

then for every $R>0$,

$$
\mathbb{P}\left(\sup _{t \geq 0}\|S \diamond G(t)\| \geq \lambda\right) \leq 2 \exp \left(-\frac{\lambda^{2}}{2 e M C_{1}^{2} B_{q}^{2}}\right),
$$

where $C_{1}$ is as in Theorem 1.1 and $B_{q}$ only depends on $q$.

Proof. It follows from Remark 2.2 and Lemma 2.3 that $C_{p, Y} \leq C_{Y} \sqrt{p}$ for $p \geq 2$. Therefore, we can conclude that $C_{2}$ from (1.1) satisfies $C_{2}=C_{p, Y} \leq C_{Y} \sqrt{p}$ for all $p \in[2, \infty)$. Using power series argument as in [17] it follows from (1.1) that for any $\epsilon>0$

$$
\begin{aligned}
\mathbb{E} \exp \left(\epsilon \sup _{t \geq 0}\|S \diamond G(t)\|^{2}\right) & =\sum_{n \geq 0} \mathbb{E} \sup _{t \geq 0} \frac{\epsilon^{n}\|S \diamond G(t)\|^{2 n}}{n !} \leq \sum_{n \geq 0} C_{1}^{2 n} C_{2}^{2 n} M^{n} \frac{\epsilon^{n}}{n !} \\
& =\sum_{n \geq 0} C_{1}^{2 n} C_{Y}^{2 n}(2 n)^{n} M^{n} \frac{\epsilon^{n}}{n !} \leq \sum_{n \geq 0} C_{1}^{2 n} C_{Y}^{2 n} 2^{n} M^{n} \epsilon^{n} e^{n}=: I,
\end{aligned}
$$

where we used $n ! \geq n^{n} e^{-n}$ in the last step. Clearly, the above expression $I=2$ for $\epsilon=2^{-1} e^{-1} M^{-1} C_{1}^{-2} C_{Y}^{-2}$. The exponential estimate now follows from:

$$
\begin{aligned}
\left.\mathbb{P}\left(\sup _{t \geq 0}\|S \diamond G(t)\|\right) \geq \lambda\right) & =\mathbb{P}\left(\exp \left(\epsilon \sup _{t \geq 0}\|S \diamond G(t)\|^{2}\right) \geq \exp \left(\epsilon \lambda^{2}\right)\right) \\
& \leq e^{-\epsilon \lambda^{2}} \mathbb{E} \exp \left(\epsilon \sup _{t \geq 0}\|S \diamond G(t)\|^{2}\right) \leq 2 e^{-\epsilon \lambda^{2}} .
\end{aligned}
$$

In the next section we present an entirely different approach to exponential tail estimates based on [6], which has the advantage that we do not need to have optimal estimates as $p \rightarrow \infty$. A disadvantage is that it is more difficult and the constants in the exponential estimate are less explicit.

\section{Alternative approach to Theorem 1.1}

In this section we present an alternative approach to obtain a version of Theorem 1.1 with a slightly different assumption on the geometry of the Banach space $X$ taken from [6]. For $r \in[2, \infty)$ consider the following condition on $X$ :

$\left(\mathrm{C}_{r}\right)$ The function $\phi: X \rightarrow \mathbb{R}$ defined by $\phi(x)=\|x\|^{r}$ is two times continuously Fréchet differentiable and there are constants $k_{1}, k_{2}>0$ such that

$$
\left\|\phi^{\prime}(x)\right\| \leq k_{1}\|x\|^{r-1} \text { and }\left\|\phi^{\prime \prime}(x)\right\| \leq k_{2}\|x\|^{r-2} .
$$

If $\left(\mathrm{C}_{r}\right)$ holds for some $r \in[2, \infty)$, then one can show that $\left(\mathrm{C}_{s}\right)$ holds for all $s \geq r$. In particular, for $X=L^{p}$ with $p \in[2, \infty),\left(\mathrm{C}_{r}\right)$ holds for all $r \in[p, \infty)$. Furthermore let us note that $\left(\mathrm{C}_{2}\right)$ can only hold for spaces which are isomorphic to a Hilbert space (see [12, Fact 1.0 in V.I]). In particular, $\left(\mathrm{C}_{2}\right)$ does not hold for any $X=L^{p}$ with $p \in(2, \infty)$. 
These estimates (3.1) are the ones used in [6] in order to obtain results on pathcontinuity under the additional assumption that $S(t)$ is a contraction semigroup.

The following result will allow us to relate our setting Theorem 1.1 to the setting in 6 .

Proposition 3.1. Let $X$ be a Banach space which satisfies $\left(C_{r}\right)$ for some $r \in$ $[2, \infty)$. Assume $A$ satisfies hypothesis $(H)$. Then there exists an equivalent norm $\|\cdot\|$ on $X$ for which $X$ also satisfies $\left(C_{r}\right)$ with the same constants, and $S(t)$ is a contraction semigroup.

Proof. By hypothesis $(H)$ and [19] we can define the following equivalent norm on $X$ :

$$
\|x\|=\left\|t \mapsto(-A)^{1 / 2} S(t) x\right\|_{\gamma_{r}\left(\mathbb{R}_{+} ; X\right)} .
$$

Here for $r=2, \gamma_{2}\left(\mathbb{R}_{+} ; X\right)=\gamma\left(\mathbb{R}_{+} ; X\right)$ is as in [26, 27]. For $r \in(2, \infty), \gamma_{r}\left(\mathbb{R}_{+} ; X\right)$ is defined using $L^{r}(\Omega ; X)$-norms of the corresponding Gaussian sums (which are all equivalent by the Kahane-Khintchine inequalities). Here $(\Omega, \mathcal{A}, \mathbb{P})$ is a probability space. We claim that $L^{r}(\Omega ; X)$ satisfies $\left(\mathrm{C}_{r}\right)$ with the same constants $k_{1}$ and $k_{2}$. Indeed, one can show that the function $\psi: X \rightarrow \mathbb{R}$ given by $\psi(y)=\|y\|_{L^{r}(\Omega ; X)}^{r}$ satisfies

$$
\begin{aligned}
\psi^{\prime}(y) v & =\int_{\Omega} \phi^{\prime}(y(\omega)) u(\omega) d \mathbb{P}(\omega), \quad u \in L^{r}(\Omega ; X), \\
\psi^{\prime \prime}(y)(u, v) & =\int_{\Omega} \phi^{\prime \prime}(y(\omega))(u(\omega), v(\omega)) d \mathbb{P}(\omega), \quad u, v \in L^{r}(\Omega ; X) .
\end{aligned}
$$

Now the claim follows from Hölder's inequality and the assumption on $X$.

As $\gamma_{r}\left(\mathbb{R}_{+} ; X\right)$ is a closed subspace of $L^{r}(\Omega ; X)$, it also satisfies $\left(\mathrm{C}_{r}\right)$ with constants $k_{1}$ and $k_{2}$. Finally, $S$ is a contraction semigroup in $(X,\|\cdot\|)$, since

$$
\begin{aligned}
\|S(s) x\| & =\left\|t \mapsto(-A)^{1 / 2} S(t+s) x\right\|_{\gamma_{r}\left(\mathbb{R}_{+} ; X\right)} \\
& =\left\|t \mapsto(-A)^{1 / 2} S(t) x\right\|_{\gamma_{r}([s, \infty) ; X)} \leq\|x\|,
\end{aligned}
$$

where we used the left ideal property in $\gamma_{r}\left(\mathbb{R}_{+} ; X\right)$ in the last line.

As a consequence we obtain the following result.

Theorem 3.2. Let $X$ be a Banach space with satisfies condition $\left(C_{r}\right)$, Assume condition $(H)$ holds. Then for all $G \in L_{\mathscr{F}}^{0}\left(\Omega ; L^{2}\left(\mathbb{R}_{+} ; \gamma(H, X)\right)\right)$ the process $S \diamond G$ has a version with continuous paths. Moreover, for all $p \in(0, \infty)$, the following maximal estimate holds:

$$
\left(\mathbb{E} \sup _{t \geq 0}\|S \diamond G(t)\|^{p}\right)^{1 / p} \leq C_{1} C_{2}\left(\mathbb{E}\|G\|_{L^{2}\left(\mathbb{R}_{+} ; \gamma(H, X)\right)}^{p}\right)^{1 / p},
$$

where $C_{1}$ depends on $A$, and $C_{2}$ depends on $X$ and $p$.

Proof. First let $p \geq r$. Then $X$ satisfies $\left(\mathrm{C}_{p}\right)$. By Proposition 3.1 we can find an equivalent norm $\|\cdot\|$ on $X$ which satisfies $\left(\mathrm{C}_{p}\right)$ and for which $S$ is a contraction semigroup. Let $b, B>0$ be such that $b\|x\| \leq\|x\| \leq B\|x\|$. By [6, Theorem 1.1] we obtain a version with continuous paths. Moreover, by [6, (1.2)] we can find a constant $K$ depending on the constants in (3.1) and $p$ such that

$$
\begin{aligned}
\left(\mathbb{E} \sup _{t \geq 0}\|S \diamond G(t)\|^{p}\right)^{1 / p} & \leq B\left(\mathbb{E} \sup _{t \geq 0}\|S \diamond G(t)\|^{p}\right)^{1 / p} \\
& \leq B K\left(\mathbb{E}\|G\|_{L^{2}\left(\mathbb{R}_{+} ; \gamma(H, X)\right)}^{p}\right)^{1 / p}
\end{aligned}
$$




$$
\leq B K b^{-1}\left(\mathbb{E}\|G\|_{L^{2}\left(\mathbb{R}_{+} ; \gamma(H, X)\right)}^{p}\right)^{1 / p}
$$

This proves the result with $C_{1}=B b^{-1}$ and $C_{2}=K$ for $p \geq r$. For $0<p<r$, the result follows from a standard application of Lenglart's stopping time argument (see [22]).

One drawback of the above approach is that the constant $C_{2}$ that comes from the proof of [6. Theorem 1.1] is somewhat complicated and probably not optimal. On the other hand by [6. Theorem 1.2] we immediately get exponential estimates.

Theorem 3.3. Let $X$ be a Banach space with satisfies condition $\left(C_{r}\right)$, Assume condition $(H)$ holds. If $G \in L_{\mathscr{F}}^{0}\left(\Omega ; L^{2}\left(\mathbb{R}_{+} ; \gamma(H, X)\right)\right)$ is such that for some $M>0$, almost surely

$$
\|G\|_{L^{2}\left(\mathbb{R}_{+} ; \gamma(H, X)\right)} \leq \sqrt{M}
$$

then for every $R>0$,

$$
\mathbb{P}\left(\sup _{t \geq 0}\|S \diamond G(t)\| \geq R\right) \leq 3 \exp \left(-\frac{R^{2}}{C_{1} C_{2} M}\right) .
$$

where $C_{1}$ depends on $A$ and $C_{2}$ depends on $X$.

Proof. Let $\|\cdot\|$ be as in the proof of Theorem 3.2 . We write $\tilde{X}$ for $X$ with the norm $\|\cdot\|$. Then

$$
\|G\|_{L^{2}\left(\mathbb{R}_{+} ; \gamma(H, \tilde{X})\right)}^{2} \leq b^{-2}\|G\|_{L^{2}\left(\mathbb{R}_{+} ; \gamma(H, X)\right)}^{2} \leq b^{-2} M .
$$

Therefore, [6. Theorem 1.2] implies that there is a constant $K>0$ depending on $p$ and $X$ such that

$$
\begin{aligned}
\mathbb{P}\left(\sup _{t \geq 0}\|S \diamond G\| \geq R\right) & \leq \mathbb{P}\left(\sup _{t \geq 0}\|S \diamond G(t)\| \geq R / B\right) \\
& \leq 3 \exp \left(-\frac{R^{2}}{B^{2} b^{-2} K M}\right) .
\end{aligned}
$$

The result follows with $C_{1}=B^{2} b^{-2}$ and $C_{2}=K$.

\section{Extensions of the Results for SpaCes With PRoperty $(\alpha)$}

In this section we present a result which does not require the type 2 assumption on the Banach space $X$. However, we do assume $X$ is a UMD space. In this setting the space of integrable processes is described by the space $L_{\mathscr{F}}^{0}\left(\Omega ; \gamma\left(\mathbb{R}_{+} ; H, X\right)\right)$ and one has the following (see [26] for details):

Proposition 4.1. 26, Theorems 5.9, 5.12] Let $E$ be a UMD Banach space and let $p \in(0, \infty)$ be fixed. For an adapted process $\Phi: \mathbb{R}_{+} \times \Omega \rightarrow \mathscr{L}(H, X)$ the following are equivalent:

(1) The process $\Phi$ is stochastically integrable with respect to $W_{H}$.

(2) $\Phi(\cdot, \omega) \in \gamma\left(\mathbb{R}_{+} ; H, X\right)$ for a.e. $\omega \in \Omega$.

In this situation we have $t \mapsto \int_{0}^{t} \Phi d W_{H}$ is a.s. pathwise continuous. Furthermore, for all $p \in(0, \infty)$, there exists constants $c_{p, X}^{\gamma}, C_{p, X}^{\gamma}>0$ such that

$$
c_{p, X}^{\gamma} \mathbb{E}\|\Phi\|_{\gamma(0, T ; H, X)}^{p} \leq \mathbb{E} \sup _{t \in[0, T]}\left\|\int_{0}^{t} \Phi d W_{H}\right\|^{p} \leq C_{p, X}^{\gamma} \mathbb{E}\|\Phi\|_{\gamma(0, T ; H, X)}^{p} .
$$


The case $0<p \leq 1$ was not considered in [26], but can easily be obtained by an application of Lenglart's inequality.

In the following result we need that the Banach space $X$ has the so-called property $(\alpha)$ (see 31 for details). Examples of UMD spaces with property $(\alpha)$ are $X=L^{q}$ with $q \in(1, \infty)$ or any space which is isomorphic to a closed subspace of $L^{q}$ with $q \in(1, \infty)$.

Theorem 4.2. Let $X$ be a UMD space with property $(\alpha)$. Assume A satisfies hypothesis $(H)$. Then for all $G \in L_{\mathscr{F}}^{0}\left(\Omega ; \gamma\left(\mathbb{R}_{+} ; H, X\right)\right)$ the process $S \diamond G$ has a version with continuous paths. Moreover, for all $p \in(0, \infty)$, the following maximal estimate holds:

$$
\left(\mathbb{E} \sup _{t \geq 0}\|S \diamond G(t)\|^{p}\right)^{1 / p} \leq C_{1} C_{2}\left(\mathbb{E}\|G\|_{\gamma\left(\mathbb{R}_{+} ; H, X\right)}^{p}\right)^{1 / p},
$$

where $C_{1}$ depends on $A$ and $w$, and $C_{2}$ depends on $X$ and $p$.

In the case that $X$ has type 2 and property $(\alpha)$ the assertion in Theorem 4.2 is stronger than Theorem 1.1. Indeed, this follows from the fact that for type 2 spaces $X$ the space $\gamma\left(\mathbb{R}_{+} ; H, X\right)$ is larger than $L^{2}\left(\mathbb{R}_{+} ; \gamma(H, X)\right)$ (see [28, 32]).

Theorem 4.2 applies to the same situation as in Example 1.2 .

Example 4.3. Let $q \in(1, \infty)$. Let $A$ and $X$ be as in Example 1.2, Then as before $A-w$ satisfies $(H)$ for some $w \in R$ large enough. Therefore, Theorem 4.2 is applicable for any $q \in(1, \infty)$. Moreover, even for $q \in[2, \infty)$ the assertion of Theorem 4.2 leads to stronger results in this example.

The proof of Theorem 4.2 is more involved. We need to apply property $(\alpha)$ to have better structural properties of the group used in the dilation argument.

Proof of Theorem 4.2. Let $Y, J \in \mathscr{L}(X, Y), P \in \mathscr{L}(Y)$ and $(U(t))_{t \in \mathbb{R}}$ in $\mathscr{L}(Y)$ be as in the proof of Theorem 1.1. The equality (2.2) still holds. However, we need some arguments to see that the stochastic integral $\int_{0}^{t} U(-s) J G(s) d W_{H}(s)$ exists in $Y$. Indeed, note that $s \mapsto U(-s) J G(s)$ is strongly measurable and adapted. Recall from [13 that $U(r) \in \mathscr{L}(Y)$ is the tensor extension (in the sense of [19]) of the usual right-translation operator on $L^{2}(\mathbb{R} ; H)$. Since $X$ has property $(\alpha)$, it follows from [14, Theorem 3.18] that $(U(r))_{r \in \mathbb{R}} \subseteq \mathscr{L}(Y)$ is $\gamma$-bounded by some constant $\alpha_{X}$. Now the multiplier result of 19 shows that $s \mapsto U(-s) J G(s)$ is in $\gamma\left(\mathbb{R}_{+} ; H ; X\right)$ a.s., and

$$
\begin{aligned}
\|U(-s) J G(s)\|_{\gamma\left(\mathbb{R}_{+} ; H, Y\right)} & \leq \alpha_{X}\|J G(s)\|_{\gamma\left(\mathbb{R}_{+} ; H, Y\right)} \\
& \leq \alpha_{X} C\|G\|_{\gamma\left(\mathbb{R}_{+} ; H ; X\right)}<\infty \text { a.s. }
\end{aligned}
$$

where the last step follows from the left-ideal property. Since $Y$ has UMD by Lemma 2.3, it follows from Proposition 4.1 that $t \mapsto \int_{0}^{t} U(-s) J G(s) d W_{H}(s)$ exists and has a version which is a.s. pathwise continuous. Therefore, by (2.2) and the strong continuity of $U(t)$ it follows that $J S \diamond G$ has a version which is a.s. pathwise continuous. As in the proof of Theorem 1.1 by (i) also $S \diamond G$ has a version which is a.s. pathwise continuous. Moreover, if $G \in L^{p}\left(\Omega ; \gamma\left(\mathbb{R}_{+} ; H, X\right)\right)$ we can use (2.2), Proposition 4.1 and (4.2) to obtain the following estimate

$$
\begin{aligned}
\mathbb{E}\left(\sup _{t \geq 0}\|S \diamond G(t)\|^{p}\right) & \leq c^{-p} \mathbb{E}\left(\sup _{t \geq 0}\|J S \diamond G(t)\|_{Y}^{p}\right) \\
& =c^{-p} \mathbb{E}\left(\sup _{t \geq 0}\left\|P U(t) \int_{0}^{t} U(-s) J G(s) d W_{H}(s)\right\|_{Y}^{p}\right)
\end{aligned}
$$




$$
\begin{aligned}
& \leq c^{-p}\|P\|^{p} \mathbb{E}\left(\sup _{t \geq 0}\left\|\int_{0}^{t} U(-s) J G(s) d W_{H}(s)\right\|_{Y}^{p}\right) \\
& \leq c^{-p}\|P\|^{p}\left(C_{p, Y}^{\gamma}\right)^{p}\|U(-s) J G(s)\|_{L^{p}\left(\Omega ; \gamma\left(\mathbb{R}_{+} ; H, X\right)\right)}^{p} \\
& \leq c^{-p}\|P\|^{p}\left(C_{p, Y}^{\gamma}\right)^{p} \alpha_{X}^{p} C^{p}\|G\|_{L^{p}\left(\Omega ; \gamma\left(\mathbb{R}_{+} ; H, X\right)\right)}^{p}
\end{aligned}
$$

This completes the proof of (1.1) with $C_{1}=c^{-1} C\|P\|$ and $C_{2}=C_{p, Y}^{\gamma} \alpha_{X}$.

At this moment we do not know if there are exponential tail estimates in the general setting of Theorem 4.2. However, also in this setting there is some hope that $C_{p, Y}^{\gamma} \leq C_{X} \sqrt{p}$ for $p$ large, and by the argument in Theorem 2.4 this would yield exponential tail estimates again. Recently, in [8] it has been proved that $C_{p, Y}^{\gamma} \leq C_{X} p$ for $p$ large. This yields exponential estimates, but no exponential quadratic estimates as one would expect.

\section{Appendix A. Optimal Constants in the Burkholder-Davis-Gundy INEQUALITY FOR STOCHASTIC INTEGRALS}

For a Banach space $X$ and $p \in(0, \infty)$ let $K_{p, X}$ be the smallest constant $K$ such that

$$
\sup _{t \geq 0} \mathbb{E}\left(\left\|\int_{0}^{t} G(s) d W_{H}(s)\right\|^{p}\right) \leq K^{p}\|G\|_{L^{p}\left(\Omega ; L^{2}\left(\mathbb{R}_{+} ; \gamma(H, X)\right)\right)}^{p},
$$

for all $G \in L_{\mathscr{F}}^{p}\left(\Omega ; L^{2}\left(\mathbb{R}_{+} ; \gamma(H, X)\right)\right)$. If there does not exist such a constant $K$, we set $K_{p, X}=\infty$. Recall from Proposition 2.1 that A.1 holds for some $K$ if $X$ is a UMD space with type 2. Moreover, in [33] it has been proved that $K_{p, X} \leq K_{X} \sqrt{p}$ for $p \geq 2$ (also see Remark 2.2). Below we provide an alternative proof of this fact for the case $X=L^{q}$ with $q \in[2, \infty)$. Also recall from real stochastic analysis that there is a constant $b>0$ such that for all $p \geq 2, K_{p, \mathbb{R}} \leq b \sqrt{p}$ (see [10]).

Proposition A.1. Let $X_{0}$ and $X_{1}$ be Banach spaces for which (A.1) holds and which form an interpolation couple. Assume $X_{0}$ is reflexive. Then the complex interpolation spaces $X_{\theta}=\left[X_{0}, X_{1}\right]_{\theta}$ with $\theta \in[0,1]$ satisfies (A.1) with

$$
K_{p, X_{\theta}} \leq K_{p, X_{0}}^{1-\theta} K_{p, X_{1}}^{\theta} .
$$

Proof. One easily checks that A.1 implies that $X_{1}$ and $X_{2}$ have type 2 (see 32]). Therefore, $X_{1}$ and $X_{2}$ are $K$-convex and this implies $\left[\gamma\left(H, X_{0}\right), \gamma\left(H, X_{1}\right)\right]_{\theta}=$ $\gamma\left(H, X_{\theta}\right)$ (see [18, Proposition 2.3] or [34]))

Fix $t \in \mathbb{R}_{+}$and let $Y=L_{\mathscr{F}}^{p}\left(\Omega ; L^{2}(0, t)\right)$. Clearly, $Y$ is Banach function space. As in 7] write $Y(X)$ for the $X$-valued strongly measurable and adapted processes $g$ with values in $X$ for which $\|g\|_{Y(X)}:=\|\| g\left\|_{X}\right\|_{Y}<\infty$. We claim that $Y\left(\gamma\left(H, X_{0}\right)\right)$ is reflexive. Indeed, note that $\gamma\left(H, X_{0}\right)$ is isometric to a closed subspace of $L^{2}\left(\tilde{\Omega} ; X_{0}\right)$ for some probability space $(\tilde{\Omega}, \tilde{\mathscr{F}}, \tilde{\mathbb{P}})$, and the latter is reflexive since $X_{0}$ is reflexive. Therefore, $\gamma\left(H, X_{0}\right)$ is reflexive as well. Now the claim follows from the fact that $Y\left(\gamma\left(H, X_{0}\right)\right)$ is a closed subspace of the reflexive space $L^{p}\left(\Omega ; L^{2}\left(0, t ; \gamma\left(H, X_{0}\right)\right)\right)$. By [7, 13.5] we obtain

$$
\left[Y\left(\gamma\left(H, X_{0}\right)\right), Y\left(\gamma\left(H, X_{1}\right)\right)\right]_{\theta}=Y\left(\left[\gamma\left(H, X_{0}\right), \gamma\left(H, X_{1}\right)\right]_{\theta}\right)=Y\left(\gamma\left(H, X_{\theta}\right)\right) .
$$

Similarly, one has

$$
\left[L^{p}\left(\Omega ; X_{0}\right), L^{p}\left(\Omega ; X_{1}\right)\right]_{\theta}=L^{p}\left(\Omega ; X_{\theta}\right) .
$$


Let $T: Y\left(\gamma\left(H, X_{i}\right)\right) \rightarrow L^{p}\left(\Omega ; X_{i}\right)$ be defined by $T G=\int_{0}^{t} G(s) d W_{H}(s)$. Then $\|T\|_{Y\left(\gamma\left(H, X_{i}\right)\right) \rightarrow L^{p}\left(\Omega ; X_{i}\right)} \leq K_{p, X_{i}}$ for $i=1,2$. Consequently, since $[\cdot, \cdot]_{\theta}$ is an (exact) interpolation method (see [35, Theorem 1.9.3]), we obtain

$$
\|T\|_{Y\left(\gamma\left(H, X_{\theta}\right)\right) \rightarrow L^{p}\left(\Omega ; X_{\theta}\right)} \leq K_{p, X_{0}}^{1-\theta} K_{p, X_{1}}^{\theta} .
$$

Since $t \in \mathbb{R}_{+}$was arbitrary, we obtain $K_{p, X_{\theta}} \leq K_{p, X_{0}}^{1-\theta} K_{p, X_{1}}^{\theta}$.

Lemma A.2. Let $p \in[2, \infty)$. The following assertions hold:

(1) If $(\mathcal{O}, \Sigma, \mu)$ be a (nonempty) $\sigma$-finite measure space, then $K_{p, L^{p}(\mathcal{O})}=K_{p, \mathbb{R}}$.

(2) If $X$ is a Hilbert space with nonzero dimension, then $K_{p, X}=K_{p, \mathbb{R}}$.

Proof.

(1): This follows from Fubini theorem. However, due to operator valued setting some technicalities have to be overcome. Write $X=L^{p}(\mathcal{O})$. By a density argument, it suffices to consider adapted step processes $G$ which take values in the finite rank operators, i.e.

$$
G=\sum_{n=1}^{N} \mathbf{1}_{\left(t_{n-1}, t_{n}\right]} \sum_{m=1}^{M} \mathbf{1}_{A_{m n}} \sum_{j=1}^{J} h_{j} \otimes x_{j m n} .
$$

Here $0=t_{0}<t_{1}<\ldots<t_{N}=t$, the sets $\left(A_{m n}\right)_{m=1}^{M}$ are in $\mathscr{F}_{t_{n}},\left(h_{j}\right)_{j=1}^{J}$ in $H$ are orthonormal and $\left(x_{j m n}\right)_{j, m, n}$ are in $X$.

Let $g: \mathbb{R}_{+} \times \Omega \times \mathcal{O} \rightarrow H$ be given by

$$
g=\sum_{n=1}^{N} \mathbf{1}_{\left(t_{n-1}, t_{n}\right]} \sum_{m=1}^{M} \mathbf{1}_{A_{m n}} \sum_{j=1}^{J} x_{j m n} \otimes h_{j} .
$$

Now fix some time $t>0$. Recall that $\gamma(H, \mathbb{R})=H$ By Fubini's theorem we can write

$$
\begin{aligned}
{\left[\mathbb { E } \left(\left\|\int_{0}^{t} G(s) d W_{H}(s)\right\|_{X}^{p}\right.\right.} & =\int_{\mathcal{O}} \mathbb{E}\left|\int_{0}^{t} g(s, \cdot, r) d W_{H}(s)\right|^{p} d \mu(r) \\
& \leq K_{p, \mathbb{R}}^{p} \int_{\mathcal{O}}\|g(\cdot, \cdot, r)\|_{L^{p}\left(\Omega ; L^{2}(0, t ; H)\right)}^{p} d \mu(r) \\
& =K_{p, \mathbb{R}}^{p}\|g\|_{L^{p}\left(\Omega ; L^{p}\left(\mathcal{O} ; L^{2}(0, t ; H)\right)\right)}^{p} \\
& \stackrel{(i)}{\leq} K_{p, \mathbb{R}}^{p}\|g\|_{L^{p}\left(\Omega ; L^{2}\left(0, t ; L^{p}(\mathcal{O} ; H)\right)\right)}^{p} \\
& \stackrel{(i i)}{\leq}\|G\|_{L^{p}\left(\Omega ; L^{2}\left(\mathbb{R}_{+} ; \gamma(H, X)\right)\right)}
\end{aligned}
$$

The estimate (i) follows from Minkowski's inequality with exponent $p / 2$. To see that (ii) holds, let $f \in L^{p}(\mathcal{O} ; H)$ and $F \in \gamma\left(H, L^{p}(\mathcal{O})\right)$ be given by $(F h)(r)=$ $[h, f(r)]_{H}$. Let $\left(h_{j}\right)_{j \geq 1}$ be an orthonormal basis for $H$. Then by randomization and Minkowski's inequality with exponent $p / 2$, we have

$$
\begin{aligned}
\|f\|_{L^{p}(\mathcal{O} ; H)} & =\left\|\left(\sum_{j \geq 1}\left|\left[h_{j}, f\right]\right|^{2}\right)^{1 / 2}\right\|_{L^{p}(\mathcal{O})}=\left\|\left(\sum_{j \geq 1}\left|F h_{j}\right|^{2}\right)^{1 / 2}\right\|_{L^{p}(\mathcal{O})} \\
& =\left\|\sum_{j \geq 1} \gamma_{j} F h_{j}\right\|_{L^{p}\left(\mathcal{O} ; L^{2}(\widetilde{\Omega})\right)} \leq\left\|\sum_{j \geq 1} \gamma_{j} F h_{j}\right\|_{L^{2}\left(\widetilde{\Omega} ; L^{p}(\mathcal{O})\right)}=\|F\|_{\gamma\left(H, L^{p}(\mathcal{O})\right)} .
\end{aligned}
$$

Here $\left(\gamma_{j}\right)_{j \geq 1}$ is a Gaussian sequence on a probability space $(\widetilde{\Omega}, \widetilde{\mathcal{A}}, \widetilde{\mathbb{P}})$. This proves (ii) and therefore, $K_{p, X} \leq K_{p, \mathbb{R}}$. The converse estimate is trivial. 
(2): This seems to be well-known to experts. A short proof can be given using (1). Fix $G \in L_{\mathcal{F}}^{p}\left(\Omega ; L^{2}\left(\mathbb{R}_{+} ; \gamma(H, X)\right)\right.$. Since $G$ is strongly measurable it takes values in a separable subspace of $\gamma(H, X)$. Thereforem we can replace $X$ by a separable Hilbert space $X_{0}$ if necessary. Now the result follows from (1), because any separable Hilbert space is isometric to a closed subspace of $L^{p}(0,1)$ (see [1, Proposition 6.4.13]).

As a consequence we obtain the following result.

Theorem A.3. Let $(\mathcal{O}, \Sigma, \mu)$ be a (nonempty) $\sigma$-finite measure space and let $q \in$ $[2, \infty)$. Let $X$ be a closed subspace of $L^{q}(\mathcal{O})$. Then for all $p \in[q, \infty)$ one has $K_{p, X}=K_{p, \mathbb{R}}$ for the optimal constants from (A.1).

Proof. Without loss of generality we can assume $X=L^{q}(\mathcal{O})$. Let $\theta \in(0,1)$ be such that $\frac{1}{q}=\frac{1-\theta}{2}+\frac{\theta}{p}$. Then it follows from Proposition A.1 with $X_{0}=L^{2}(\mathcal{O})$ and $X_{1}=L^{p}(\mathcal{O})$ and $X_{\theta}=L^{q}(\mathcal{O})$ that $K_{p, L^{q}(\mathcal{O}} \leq K_{p, L^{2}(\mathcal{O})}^{1-\theta} K_{p, L^{p}(\mathcal{O})}^{\theta}$. Combining this with Lemma A.2 yields $K_{p, L^{q}(\mathcal{O})} \leq K_{p, \mathbb{R}}$. The converse inequality is trivial.

Corollary A.4. Let $(\mathcal{O}, \Sigma, \mu)$ be a (nonempty) $\sigma$-finite measure space, let $q \in$ $[2, \infty)$ and let $X$ be a closed subspace of $L^{q}(\mathcal{O})$. Then for all $p \in[q, \infty)$,

$$
\left(\mathbb{E} \sup _{t \geq 0}\left\|\int_{0}^{t} G(s) d W_{H}(s)\right\|_{X}^{p}\right)^{1 / p} \leq K_{p, \mathbb{R}} p^{\prime}\|G\|_{L^{p}\left(\Omega ; L^{2}\left(\mathbb{R}_{+} ; \gamma(H, X)\right)\right)},
$$

where $p^{\prime} \in(1,2]$ is such that $\frac{1}{p}+\frac{1}{p^{\prime}}=1$.

Recall that there is a constant $b>0$ such that $K_{p, \mathbb{R}} \leq b \sqrt{p}$ for all $p \in[2, \infty)$. Therefore, in the above result we have $K_{p, \mathbb{R}} p^{\prime} \leq 2 b \sqrt{p}$ as soon as $p \in[q, \infty)$. This is a rather precise description of the behavior of the constant as $p \rightarrow \infty$ and has important consequences.

Proof. This follows directly from Doob's maximal $L^{p}$-inequality for the submartingale $\left\|\int_{0}^{\cdot} G(s) d W_{H}(s)\right\|$ combined with (A.3).

Acknowledgement In the first version of this paper the proof of Theorem 2.4 was only valid for the case $X=L^{q}$. The authors thank professor Jan Seidler for sending them his paper [33] where it has been proved that the constants in Proposition 2.1] behave as $C_{p, X} \leq C_{X} \sqrt{p}$ for $p$ large. This enabled us to cover all UMD spaces with type 2 in Theorem 2.4.

The authors would like to thank the anonymous referee for pointing out a mistake in Section 3 .

\section{REFERENCES}

[1] F. Albiac and N.J. Kalton. Topics in Banach space theory, volume 233 of Graduate Texts in Mathematics. Springer, New York, 2006.

[2] H. Amann. Dual semigroups and second order linear elliptic boundary value problems. Israel J. Math., 45(2-3):225-254, 1983.

[3] Z. Brzeźniak. Stochastic partial differential equations in M-type 2 Banach spaces. Potential Anal., 4(1):1-45, 1995.

[4] Z. Brzeźniak. On stochastic convolution in Banach spaces and applications. Stochastics Stochastics Rep., 61(3-4):245-295, 1997.

[5] Z. Brzeźniak and E. Hausenblas. Maximal regularity for stochastic convolutions driven by Lévy processes. Probab. Theory Related Fields, 145(3-4):615-637, 2009. 
[6] Z. Brzeźniak and S. Peszat. Maximal inequalities and exponential estimates for stochastic convolutions in Banach spaces. In Stochastic processes, physics and geometry: new interplays, I (Leipzig, 1999), volume 28 of CMS Conf. Proc., pages 55-64. Amer. Math. Soc., Providence, RI, 2000.

[7] A.-P. Calderón. Intermediate spaces and interpolation, the complex method. Studia Math., 24:113-190, 1964.

[8] S.G. Cox and M.C. Veraar. Vector-valued decoupling and the Burkholder-Davis-Gundy inequality. Submitted, 2010.

[9] G. Da Prato and J. Zabczyk. Stochastic equations in infinite dimensions, volume 44 of Encyclopedia of Mathematics and its Applications. Cambridge University Press, Cambridge, 1992.

[10] B. Davis. On the $L^{p}$ norms of stochastic integrals and other martingales. Duke Math. J., 43(4):697-704, 1976.

[11] R. Denk, G. Dore, M. Hieber, J. Prüss, and A. Venni. New thoughts on old results of R. T. Seeley. Math. Ann., 328(4):545-583, 2004.

[12] R. Deville, G. Godefroy, and V. Zizler. Smoothness and renormings in Banach spaces, volume 64 of Pitman Monographs and Surveys in Pure and Applied Mathematics. Longman Scientific \& Technical, Harlow, 1993.

[13] A.M. Fröhlich and L.W. Weis. $H^{\infty}$ calculus and dilations. Bull. Soc. Math. France, 134(4):487-508, 2006.

[14] B.H. Haak and P.C. Kunstmann. Admissibility of unbounded operators and wellposedness of linear systems in Banach spaces. Integral Equations Oper. Theory, 55(4):497-533, 2006.

[15] M. Haase. The functional calculus for sectorial operators, volume 169 of Operator Theory: Advances and Applications. Birkhäuser Verlag, Basel, 2006.

[16] E. Hausenblas and J. Seidler. A note on maximal inequality for stochastic convolutions. Czechoslovak Math. J., 51(126)(4):785-790, 2001.

[17] E. Hausenblas and J. Seidler. Stochastic convolutions driven by martingales: maximal inequalities and exponential integrability. Stoch. Anal. Appl., 26(1):98-119, 2008.

[18] T. Hytönen, J. van Neerven, and P. Portal. Conical square function estimates in UMD Banach spaces and applications to $H^{\infty}$-functional calculi. J. Anal. Math., 106:317-351, 2008.

[19] N.J. Kalton and L.W. Weis. The $H^{\infty}$-calculus and square function estimates. Preprint, 2004.

[20] P.C. Kunstmann and L.W. Weis. Maximal $L_{p}$-regularity for parabolic equations, Fourier multiplier theorems and $H^{\infty}$-functional calculus. In Functional analytic methods for evolution equations, volume 1855 of Lecture Notes in Math., pages 65-311. Springer, Berlin, 2004.

[21] M. Langer and V. Maz'ya. On $L^{p}$-contractivity of semigroups generated by linear partial differential operators. J. Funct. Anal., 164(1):73-109, 1999.

[22] E. Lenglart. Relation de domination entre deux processus. Ann. Inst. H. Poincaré Sect. B (N.S.), 13(2):171-179, 1977.

[23] V. Liskevich, Z. Sobol, and H. Vogt. On the $L_{p}$-theory of $C_{0}$-semigroups associated with second-order elliptic operators. II. J. Funct. Anal., 193(1):55-76, 2002.

[24] A. McIntosh. Operators which have an $H_{\infty}$ functional calculus. In Miniconference on operator theory and partial differential equations (North Ryde, 1986), volume 14 of Proc. Centre Math. Anal. Austral. Nat. Univ., pages 210-231. Austral. Nat. Univ., Canberra, 1986.

[25] J.M.A.M. van Neerven. $\gamma$-radonifying operators - a survey. http://arxiv.org/abs/0911.3788. 2009.

[26] J.M.A.M. van Neerven, M.C. Veraar, and L.W. Weis. Stochastic integration in UMD Banach spaces. Ann. Probab., 35(4):1438-1478, 2007.

[27] J.M.A.M. van Neerven, M.C. Veraar, and L.W. Weis. Stochastic evolution equations in UMD Banach spaces. J. Funct. Anal., 255(4):940-993, 2008.

[28] J.M.A.M. van Neerven and L.W. Weis. Weak limits and integrals of Gaussian covariances in Banach spaces. Probab. Math. Statist., 25(1, Acta Univ. Wratislav. No. 2784):55-74, 2005.

[29] I. Pinelis. Optimum bounds for the distributions of martingales in Banach spaces. Ann. Probab., 22(4):1679-1706, 1994.

[30] G. Pisier. Martingales with values in uniformly convex spaces. Israel J. Math., 20(3-4):326350, 1975.

[31] G. Pisier. Some results on Banach spaces without local unconditional structure. Compositio Math., 37(1):3-19, 1978. 
[32] J. Rosiński and Z. Suchanecki. On the space of vector-valued functions integrable with respect to the white noise. Colloq. Math., 43(1):183-201 (1981), 1980.

[33] J. Seidler. Exponential estimates for stochastic convolutions in 2-smooth Banach spaces. preprint, 2010.

[34] J. Suárez and L.W. Weis. Interpolation of Banach spaces by the $\gamma$-method. In Methods in Banach space theory, volume 337 of London Math. Soc. Lecture Note Ser., pages 293-306. Cambridge Univ. Press, Cambridge, 2006.

[35] H. Triebel. Interpolation theory, function spaces, differential operators. Johann Ambrosius Barth, Heidelberg, second edition, 1995.

[36] M.C. Veraar. Continuous local martingales and stochastic integration in UMD Banach spaces. Stochastics, 79(6):601-618, 2007.

[37] L.W. Weis. The $H^{\infty}$ holomorphic functional calculus for sectorial operators - a survey. In Partial differential equations and functional analysis, volume 168 of Oper. Theory Adv. Appl., pages 263-294. Birkhäuser, Basel, 2006.

Delft Institute of Applied Mathematics, Delft University of Technology, P.O. Box 5031, 2600 GA Delft, The Netherlands

E-mail address: M.C.Veraar@tudelft.nl

Institut für Analysis, Universität Karlsruhe (TH), D-76128 Karlsruhe, Germany

E-mail address: Lutz.Weis@math.uni-karlsruhe.de 